\title{
Space Analysis of Radio Signals ${ }^{1,2}$
}

\author{
John B. Smyth
}

(August 22, 1960)

\begin{abstract}
The radio antenna is viewed as a space frequency filter with an output just equal to the convolution of its transfer function with the radio field illuminating its aperture. An equivalent uncertainty principle limits the accuracy with which the spatial distribution of the radio field may be determined. The radio field generated by an antenna is distorted in passing into the ionosphere, generating new space frequencies which is the information contained in the field at the receiving antenna. The energy diffracted into the different orders will appear to arrive from different directions, and the angle of arrival for a given order will be a function of the radiofrequency.
\end{abstract}

\section{Introduction}

Radio fields are functions of space and time of the form

$$
f(\omega t+\bar{k} \cdot \bar{r})
$$

where $\omega$ is $2 \pi$ times the time frequency in cycles per unit time and

$$
\bar{k}=\bar{i} k_{x}+\bar{j} k_{y}+\bar{k} k_{z}
$$

is $2 \pi$ times the space frequency ${ }^{3}$ in cycles per unit distance along the given direction. A monochromatic plane wave propagating in a homogeneous isotropic medium will have space frequencies ranging from zero along directions in the plane of the wave front to a maximum equal to $2 \pi$ divided by the wave length along the direction of propagation. For directions making an angle $\theta$ with the direction of propagation, the space frequency, or wave number becomes

$$
k(\theta)=\frac{2 \pi}{\lambda} \cos \theta .
$$

If the time frequency of the plane wave is not constant, then the space frequency will not be constant along directions other than $\theta=90^{\circ}$, which corresponds to zero space frequency. Thus, a radio wave modulated in the time domain has a corresponding modulation in the space domain.

Even with a source monochromatic in the time domain, there is a spectrum of space frequencies which is generated by the radiating system, and this spectrum is a unique property of the current distribution associated with the radiating structure. In a homogeneous isotropic medium, the field everywhere is described in terms of this spectrum. In nonhomogeneous media this spectrum is continually modified as the wave propagates along, the inhomogeneities generate new space frequencies which feed

1 Contribution from Smyth Research Associates, 3555 Aero Court, San Diego 11, Calif.

2 Paper presented at Conference on Transmission Problems Related to HighFrequency Direction Finding, at UCLA, June 21-24, 1960.

Frequency Direction Finding, at UCLA, June 21-24, 1960.
3 The space frequencies are frequently called wave numbers and sometimes modes, but it is felt that the term space frequency is more appropriate in this d iscussion. on the energy of the original field. Other treatments of the effects of inhomogeneities, with special application to the ionosphere, have been reviewed by Ratcliffe [1956].

\section{Space Signals Developed Over Ionospheric Links (1.5-15 Mc/s)}

Tropospheric effects at these frequencies will be macroscopic in nature and the gross decrease in refractive index with elevation will produce a downward bending which will amount to some $22 \mathrm{~min}$ at an elevation angle of $2^{\circ}$, and $3 \mathrm{~min}$ at $20^{\circ}$. The ionosphere on the other hand, will appear dense and turbulent to the radiation propagating in the forward direction.

If, at each turning point of the radio ray, a reflecting plane parallel to the earth is assumed, and the intervening medium is considered homogeneous, then the radio field developed at the earth is just that associated with the image of the transmitter. Since the transmitter is at a large distance from the receiver, the waves arriving are almost plane and the phase wave length in a plane tangent to the earth's surface is the ratio of the radio wave length to the cosine of the elevation angle.

The medium between the reflecting plane and the earth is nonhomogeneous. In addition to the more or less regular change of electron density with elevation there are irregular patches of ionization and, in particular, filaments left in the wake of meteors at elevations between 80 and $120 \mathrm{~km}$. In propagating through these irregularities, the radio waves are distorted and the image of the transmitting antenna in the ionospheric mirror becomes blurred. There are ionospheric conditions where the ray path may indicate directions up to $180^{\circ}$ off the great circle path between the transmitter and the receiver. In any case, all that is available to the observer is the output voltage from the antenna which is generated by the radio field in its aperture. Certain deductions are made based upon the sampling of the radio field with the antenna which is nothing more than a bandpass space frequency filter. If the antenna is rotated, this is equivalent to shifting the pass-band across the space frequency domain in a given direc- 
tion. If the radio field consists of a single plane wave, the output voltage from the antenna will be its transfer function for this specific cut in the space frequency domain. For an arbitrary radio field illumination, the output from an antenna is the convolution of the radio field with its transfer function. As the antenna rotates, there may be several peaks in the output, each corresponding to increased signal derived from certain of the space frequencies making up the space signal. These results are usually interpreted as multipath transmission, where the signal is assumed to be coming from several directions.

\subsection{Radio Field Distortion in Nonhomogeneous Medium}

Returning to the assumed case of a plane reflector at the ray turning point in the ionosphere, the radio field at the receiver can be considered as produced by transmission from the image transmitter through the inhomogeneous stratum, the ionosphere. Let it be assumed that the ionosphere is plane and horizontally stratified and the transmission problem is depicted in figure 1 . If the separation of $T^{\prime}$ the transmitter image and $R$, the receiver (along the $z$ direction) is large compared with the layer thickness $2 l$, the radiation incident on the layer may be assumed plane. In this case, the solution of the field as a function of $z$ is all that is required.

A plane wave polarized normal to the incident plane will produce a field in the inhomogeneous medium of the form

$$
E_{x}(z, t)=A(z, t) \sin \left[\omega t-k_{z} z+\varphi(z, t)\right]
$$

This field satisfies the inhomogeneous wave equation

$$
\frac{d^{2} E_{x}}{d z^{2}}+k_{z}^{2} E_{x}=f\left(E_{x}, \frac{\partial E_{x}}{\partial t}\right)
$$

If it is assumed that $A$ and $\varphi$ are slowly varying functions of $z$, so that to a first approximation $f$ $\left(E_{x}, \frac{\partial E_{x}}{\partial t}\right)$ may be neglected, then the unperturbed

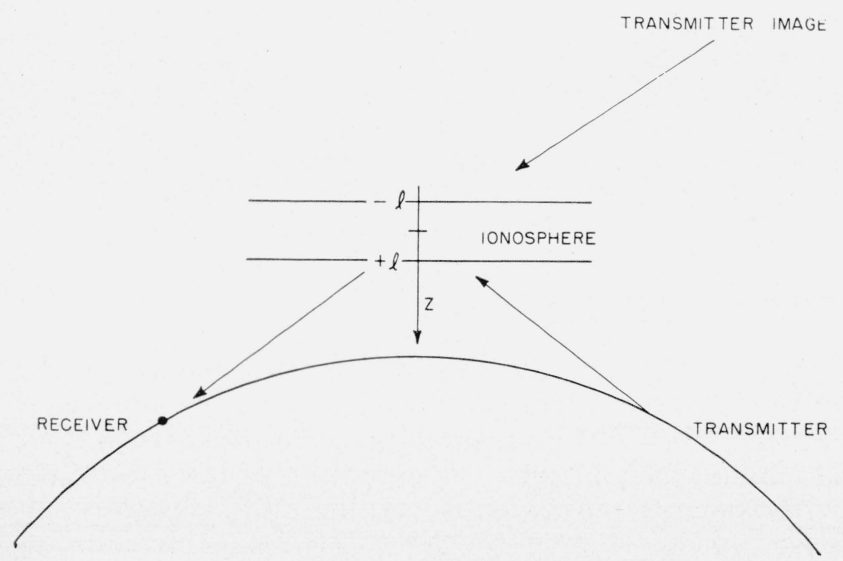

Figure 1. Ionospheric link-downcoming waves generated by horizontally stratified inhomogeneous layer. solution becomes

and

$$
E_{x}=A \sin \left(\omega t-k_{z} z+\varphi\right),
$$

$$
\frac{\partial E_{x}}{\partial z}=-A k_{z} \cos \left(\omega t-k_{z} z+\varphi\right) \text {. }
$$

Differentiating (3) with respect to $z$ and substituting for $\frac{\partial E_{x}}{\partial z}$ from (6) yields

$$
A^{\prime} \sin [\omega t-k z+\varphi]+A \varphi^{\prime} \cos [\omega t-k z+\varphi]=0 .
$$

Taking the derivative of (6) with respect to $z$ and substituting into (4) yields

$$
\begin{array}{r}
\frac{\partial A}{\partial z} k_{z} \cos [\omega t-k z+\varphi]-A k \frac{\partial \varphi}{\partial z} \sin [\omega t-k z+\varphi] \\
=f\left(E_{x}, \frac{\partial E_{x}}{\partial t}\right)
\end{array}
$$

Multiplying (7) by $\sin [\omega t-k z+\varphi]$ and (8) by $\left(\frac{1}{k}\right)$ $\cos [\omega t-k z+\varphi]$ and

adding gives

$$
\frac{\partial A}{\partial z}=\frac{1}{k} f\left(E_{x}, \frac{\partial E x}{\partial t}\right) \cos [\omega t-k z+\varphi] .
$$

In a similar manner

$$
\frac{\partial \varphi}{\partial z}=\frac{-1}{k A} f\left(E_{x}, \frac{\partial E_{x}}{\partial t}\right) \sin [\omega t-k z+\varphi] .
$$

It is assumed that the rates of change of the amplitude and phase vary little in a space period $2 \pi / k$. The mean values fo $\frac{\partial A}{\partial z}$ and $\frac{\partial \varphi}{\partial z}$ in one period are given by:

$$
\begin{gathered}
\frac{\overline{\partial A}}{\partial z}=\frac{1}{2 \pi k} \int_{0}^{2 \pi} f\left(E_{x}, \frac{\partial E_{x}}{\partial t}\right) \cos k z d(k z), \\
\frac{\partial \varphi}{\partial z}=-\frac{1}{2 \pi k A} \int_{0}^{2 \pi} f\left(E_{x}, \frac{\partial E_{x}}{\partial t}\right) \sin k z d(k z) . \\
\text { If } f\left(E_{x}, \frac{\partial E_{x}}{\partial t}\right) \text { is a function of } E_{x} \text { only, the average }
\end{gathered}
$$

change in the amplitude of the radio field over a wave length is zero. On the other hand, equation (12) does not vanish, and the phase change per wave length is the first order effect associated with the penetration of the field into the inhomogeneous medium. For the case where the dominant term in $f$ is a function of $\frac{\partial E_{x}}{\partial t}$, the variation in amplitude is the first order effect, as would be expected in cases where dissipation overrides linear and nonlinear restoring forces. 
In the case of high frequency radio wave transmission through the ionosphere, it is expected that the cases of interest are those for which the first order effect is a phase distortion. In any event, for direction finding applications, conditions which produce attenuation will reduce the space signal at the receiving antenna and limit the usefulness of this propagation mode. As the radio wave front progresses into the ionospheric layer, some parts of it are advanced in phase with respect to other parts as a result of the blobby, granular index of refraction structure of the medium. It will be assumed that the first order effect of the inhomogeneous layer on the emerging radio field is a phase distortion; which, of course, will produce an amplitude variation in the far field. An optics system is a good example of this phenomenon: a plane light wave incident upon a lens is distorted in phase but not amplitude in a plane immediately behind the lens, and this aperture field distribution creates the usual variation in intensity in the focal plane. Thus, a distortion in the phase of the radio field by the ionosphere will create new space frequencies which modulate the space signal at the earth's surface. The movements of the ionospheric irregularities will create a time and space variation of the phase distortion which in turn produces a time and space variation of the amplitude of the radio field at the surface of the earth.

In order to describe the output of the receiving antenna, it is necessary to specify the space signal in its aperture plane. The phase distortion produced by the ionosphere may be described in terms of its Fourier components. For simplicity, let it be assumed that at $z=l$ the phase distortion has a dominant component along the $\mathrm{y}$ direction with amplitude, $a$, and space frequency or wavenumber $k_{a}$. The amplitude of the radio field is assumed constant and the phase in equation (3) is given by

$$
\varphi_{z=l}(y)=a \sin k_{a} y .
$$

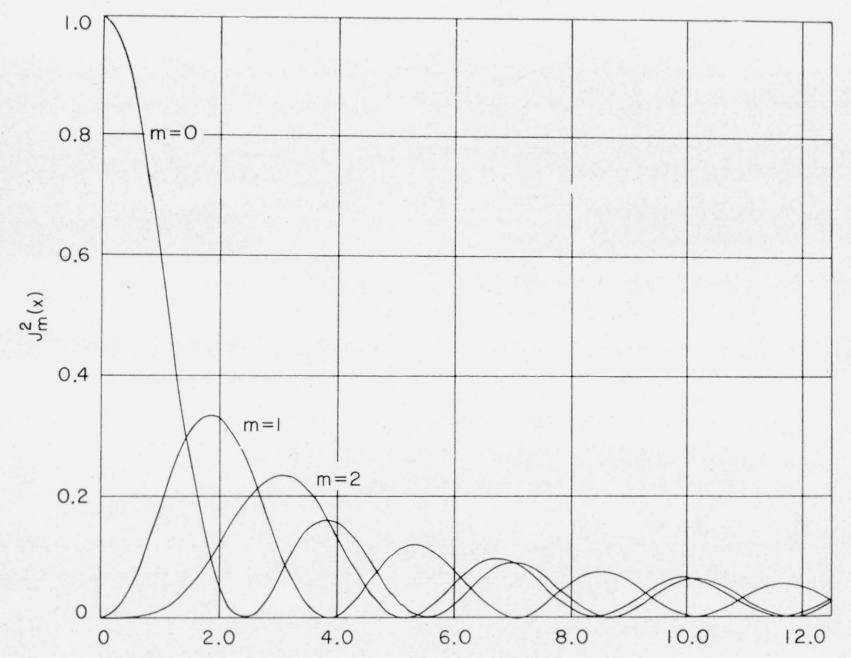

Figure 2. Energy diffracted into first three orders as a function of the radio phase distortion.
It can be shown [Smyth, 1959] that this phase distortion over a distance $y=2 b$ will diffract energy into other radio wave space frequencies with amplitude given by

$$
A\left(k_{y}\right)=\frac{b}{\pi} \sum_{-\infty}^{\infty} J_{m}(a) \frac{\sin \left(k_{y}-m k_{a}\right) b}{\left(k_{y}-m k_{a}\right) b}
$$

where $J_{m}(a)$ is the Bessel function of order $m$, and $a$, is the amplitude of the phase distortion. The radio space frequency in this case is its value along the $y$-axis, where, of course, the $\cos \theta$ in equation (2) is replaced by $\sin \theta$. If the region over which the phase distortion takes place is large compared with the radio wave frequency, then the amplitude of the new space frequencies generated by diffraction will differ from zero only in the immediate vicinity of the spectral lines

$$
k_{y m}=m k_{a}
$$

since the function

$$
\frac{\sin \left(k_{y}-m k_{a}\right) b}{\left(k_{y}-m k_{a}\right) b}
$$

is a maximum value for zero argument. The intensity of the radio field diffracted into each order is given by the square of the amplitude function, $J_{m}$ (a), which is just unity, when $m=0$ and $a=0$. 'This is the case of no distortion and for this case equation (14) reduces to a single line, $k_{y}=0$, which is just the original plane wave. It is of interest to look at equation (14) and observe the effect of radio frequency on the amplitude of the diffracted orders. Figure (2) gives $J_{m}^{2}(a)$ for $m=0,1,2$. It is clear that the energy diffracted out of the original plane wave never completely reappears in any one higher order. For small phase distortion we have

$$
\begin{aligned}
& J_{o}\left(\frac{2 \pi}{\lambda} L\right) \rightarrow 1, \\
& J_{1}\left(\frac{2 \pi}{\lambda} L\right) \rightarrow \frac{\pi L}{\lambda}, \\
& J_{2}\left(\frac{2 \pi}{\lambda} L\right) \rightarrow 1 / 2\left(\frac{\pi L}{\lambda}\right)^{2},
\end{aligned}
$$

where $a=(2 \pi L / \lambda)$ is the amplitude of the radio phase variation from its mean value. Capital $L$ is the deviation of the radio phase from the mean.

\section{Antenna as Space Frequency Filter}

\subsection{Antenna Response}

The output voltage of an antenna is determined by its filter characteristics and the electromagnetic field in which it is immersed. For a given orientation of an antenna, the output voltage is a singlevalued function ot the distribution of electromagnetic field over the effective aperture of the antenna, but unfortunately the converse is not true, there are an infinite number of field distributions that will yield the specified voltage output. 
Let the aperture plane of the antenna be the $x y$ plane $z=0$. If the antenna is fed radio energy at a constant time frequency, a resulting field distribution $E(x, y)$ will be maintained over the aperture plane. This aperture distribution may be represented by a spectral distribution of space frequencies in much the same way that a time signal may be represented in the time frequency domain. In terms of plane wave functions, the space representation of the field is given in the space frequency domain by the three dimensional Fourier transform:

$$
\begin{aligned}
& A\left(k_{x}, k_{y}, k_{z}\right)=\int_{-\infty}^{\infty} \int_{-\infty}^{\infty} \int_{-\infty}^{\infty} E(x, y, z) \\
& e^{+j k_{x} x+j k_{y} y+j k_{z} z} d x d y d z,
\end{aligned}
$$

where $A\left(k_{x}, k_{y}, k_{z}\right)$ is the amplitude of the plane wave associated with the direction $\left(k_{x}, k_{y}, k_{z}\right)$, and, as usual, the space frequency components in the cartesian coordinate system are related as follows:

$$
k_{x}^{2}+k_{y}^{2}+k_{z}^{2}=k^{2} .
$$

For the case where all the space frequency components are real, the fields are propagated waves and the amplitude function $A(k)$ evaluated for the field distribution over any plane is just the polar diagram of this particular radio field distribution [Booker and Clemmow, 1950].

Fields in the $x y$ plane, for which $k_{x}^{2}+k_{y}^{2}>k^{2}$ correspond to waves beyond cutoff in the $z$ direction and these make up the so-called "induction field" of the aperture distribution. The distorted radio field emanating from the ionosphere may have components in the current distribution which are of this type, in which case the antenna will be in the induction field for these components. At the distance involved, only the spectral components of the induction field in the immediate region of cutoff will be felt at the ground, since the higher space frequencies will be attenuated rapidly with distance.

Considering the field in the aperture plane $z=0$, the space frequency representation of the radio field and the field distribution in space are related by the two-dimensional Fourier transform parr

$$
\begin{aligned}
& A\left(k_{x}, k_{y}\right)=\iint E(x, y) e^{j k_{x} x+j k_{y}} d x d y, \\
& E(x, y)=\iint A\left(k_{x}, k_{y}\right) e^{-j k_{x} x-j k_{y} y} d k_{x} d k_{y} .
\end{aligned}
$$

The output of an antenna placed in this field distribution will simply be the superposition of the responses to the various plane wave components in the field illuminating the antenna aperture. In terms of the space frequencies, the space signal is just

$$
A\left(k_{x}, k_{y}\right)=\iint A\left(k_{x}, k_{y}\right) \delta\left(k_{x}-k_{x}^{\prime}, k_{y}-k_{y}^{\prime}\right) d k_{x}^{\prime} d k_{y}^{\prime},
$$

where $\delta$ is the impulse function in the space frequency domain; that is, a plane wave of unit strength arriving from some direction $\bar{k}$ with the property

$$
\begin{aligned}
\delta\left(k_{x}-k_{x}^{\prime}, k_{y}-k_{y}^{\prime}\right)=1 & k_{x}^{\prime}=k_{x} \\
& k_{y}^{\prime}=k_{y} \\
=0 & k_{x}^{\prime} \neq k_{x} \\
& k_{y}^{\prime} \neq k_{y} .
\end{aligned}
$$

Let the impulse response of the antenna be $G\left(k_{x}, k_{y}\right)$, then the output will be the sum of the responses created by the input signal and may be written thus:

$$
V\left(k_{x}, k_{y}\right)=\iint G\left(k_{x}-k_{x}^{\prime}, k_{y}-k_{y}^{\prime}\right) A\left(k_{x}^{\prime}, k_{y}^{\prime}\right) d k_{x}^{\prime} d k_{y}^{\prime},
$$

or,

$$
V\left(k_{x}, k_{y}\right)=\iint G\left(k_{x}^{\prime}, k_{y}^{\prime}\right) A\left(k_{x}-k_{x}^{\prime}, k_{y}-k_{y}^{\prime}\right) d k_{x}^{\prime} d k_{y}^{\prime}
$$

since the output must be the same linear combination of the displaced signal as the displaced response. Now, if the signal is the unit impulse function, the output will be just the space frequency filter response function.

$$
V\left(k_{x}, k_{y}\right)=\iint G\left(k_{x}^{\prime}, k_{y}^{\prime}\right) \delta\left(k_{x}-k_{x}^{\prime}, k_{y}-k_{y}^{\prime}\right) d k_{x}^{\prime} d k_{y}^{\prime}=G\left(k_{x}, k_{y}\right) .
$$

Since the aperture field input may be expressed as the superposition of impulse functions with appropriate amplitude and phase, the output will be the convolution of the input space frequencies and the impulse response of the antenna, which is the voltage "gain-function" of the antenna. Thus, the antenna output is uniquely given by the input signal; on the other hand, the space signal is not a single-valued function of the output.

It is clear that the output given in equation (24) will be the filter response function when the input is a plane wave function. When the input is of more complex nature, the output will not be simply related to the filter transfer function. For some unknown reason, many radio people have chosen to assume that the input signal is always of the form of a single plane wave, and that the output resulting from the convolution of the actual input signal and the filter response function of the antenna is a new response function for the antenna. This practice of absorbing the unknown aperture distribution of the radio field into the antenna filter function has led to considerable confusion, where some people have gone so far as to state that the antenna placed in a nonplane wave radio field "lost its gain." 4 A more sophisticated way of putting the same thing is the more current statement that there has developed an "antenna-to-medium coupling loss." Of course, the antenna is excited by the radio field

4 This reminds us of the small boy riding his bicycle down a rough road, concluding that each time a wheel rolled over a depression that the wheel lost its diameter. 
in its aperture and this radio field is influenced by the medium; but in electromagnetic theory the differences in physical properties of media are accounted for by charge and current distributions. Thus, the only way an antenna can be coupled to a medium is through the fields. For example, it would be of questionable value to couple to a waveguide capable of propagating many modes without regard to the mode being transmitted at the time. Even though the so-called medium remains the same independent of the mode being propagated, the fields are quite different, and the concept of antennato-medium coupling loss is of no real utility.

In general, the field distribution across the aperture of the antenna will change with time and consequently the output from the antenna most likely will change with time. Both tropospheric and ionospheric transmission studies indicate that the functional form of the aperture field, $E(x, y$,$) ,$ changes with time associated with a continual redistribution of radio energy among the different space frequencies. In direction finding experiments this corresponds to a meandering and spread in the apparent direction and location of the transmitter. Even when the propagation effects are neglected, a time modulation of the radio field will produce an uncertainty in the determination of the angle of arrival. This effect has been discussed by Dausin et al., [1959], who have shown that the error can become appreciable when the time represented by the reciprocal of the bandwidth is less than the propagation time across the antenna aperture.

\subsection{Uncertainty Principle}

If the space signal could be sampled at a point by an isotropic antenna, and even if the radio field at the point could be measured with unlimited precision, the signal variation in space or direction of arrival would be completely undetermined. To gain information on the space signal which contains information on the arrival direction requires the utilization of space. Again considering the $x y$ plane $z=0$ as the antenna aperture plane, the range of uncertainty in the space frequencies making up the signal along a given direction in the plane is related to the available aperture in that direction by the relationship

$$
\Delta|k| \cdot \delta|r| \geq \beta
$$

This is, in essence, the celebrated "Uncertainty Principle." The direction property of an antenna is a good example of this principle; if it is desired to radiate most of the energy in a specific direction then the effective aperture must be large. At first glance one might conclude that supergaining violates this relationship, but this is not the case as can be seen from the fact that the effective aperture of the antenna increases with increase in antenna gain according to the relationship (25). Diffraction of light by a slit clearly demonstrates this phenomenon. Any attempt to pass a plane wave through a confined region generates new space frequencies which spread
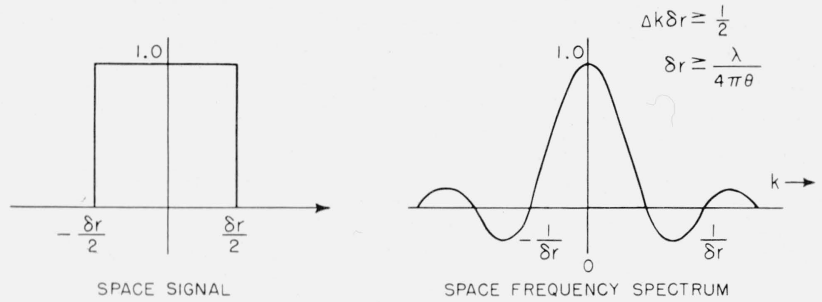

Figure 3. Constant space signal of finite extent and its space frequency spectrum.

the energy out about the original direction $\bar{k}$ in inverse relationship to the width of the slit.

Suppose that a plane wave is incident normally on the $x y$ plane and its amplitude is measured over a strip of finite width $\delta r$. If it is assumed that the space signal is zero outside this range, then the space frequency spectrum is given by

$$
C(k)=(\delta r) \frac{\sin \pi k(\delta r)}{\pi k(\delta r)} .
$$

This space signal and its spectrum is shown in figure 3. On the other hand, any continuous space signal with continuous first derivatives will appear to be constant over a space interval chosen small enough. If, in this case, the space signal is assumed to be constant throughout space, the space frequency spectrum will reduce to a single discrete line. Clearly, then, there are an infinite number of spectral distributions which would yield the same space signal over a given range. To define a spectrum uniquely, the signal must be defined over the entire $x y$ plane. Thus, given a region of space over which the signal will be sampled, (25) places a limit on the accuracy with which the space frequency may be specified.

An example of some practical interest is the space signal distortion generated by the transmission of a plane wave through an inhomogeneous medium such as the earth's atmosphere, including both the troposphere and the ionosphere. This will deteriorate further the accuracy with which the space frequency may be specified and the relationship in (25) is indeed an inequality. The question asked concerns the possibility of filtering out the space frequency noise as a means of reducing the magnitude of the product on the left side of $(25)$. If the characteristics of the space frequency noise are known, it should be possible to design optimum space frequency filters to reduce the uncertainty in the direction of arrival of the desired signal.

\section{References}

Booker, H. G., and P. C. Clemmow, The concept of an angular spectrum of plane waves and its relation to that of polar diagram and aperture distribution, Proc. IEE 97, pt. 3, 11-17 (Jan. 1950).

Dausin, L. R., K. E. Niebuhr, and J. J. Nilsson, The effects of wide-band signals on radar antenna design, IRE WESCON Conv. Record (Aug. 1959).

Ratcliffe, J. A., Some aspects of diffraction theory and their application to the ionosphere, Repts. Progr. Phys. 19, 188266 (1956).

Smyth, John B., Radio phase distortion-A mechanism for transhorizon radio propagation, URSI Washington meeting (May 1959).

(Paper 65D3-131) 